\title{
The Application of GIS-based Spatial Reasoning in the Design of Transmission Lines
}

\author{
Ping JIANG \\ Institute of geographic sciences and natural resources \\ research, CAS \\ Beijing, China \\ jiangping@1reis.ac.cn
}

\author{
Kun LI \\ Research \& development department \\ People search network co. LTD \\ Beijing, China
}

\begin{abstract}
An algorithm is proposed for precise extraction and comprehensive analysis of various complicated meteorological parameters along transmission lines. By means of GIS-based spatial reasoning, spatial relations between transmission lines and meteorological parameters were analyzed and handled properly, providing a solid foundation for the design of transmission lines. Experiments proved that the algorithm could effectively extract and analyze meteorological parameters along transmission lines, and the results were highly reliable and accurate.
\end{abstract}

Index Terms - transmission line, meteorological parameter, spatial reasoning.

\section{INTRODUCTION}

Accidents, which have significant impact on transmission lines and occur frequently, are pollution flashover, excessive icing and so forth under operating voltage at the moment $[1,2]$. Such accidents will lead directly to large-area blackouts in power grids and even result in violent collapses of regional power grids, severely affecting the safe operation of power grids and causing significant losses to China's economy. To determine meteorological conditions along schemes of transmission lines, which often requires extraction and characterization of the distribution of contamination, icing and other meteorological parameters along schemes, is one of the most important anti-pollution flashover and anti-icing work in the design of transmission lines in electric power systems. The distribution of meteorological parameters provides a solid foundation for designers, who are in charge of making configurations for anti-pollution levels and anti-icing thicknesses of transmission lines to make sure that the selected towers, conductors and insulators satisfy particular meteorological conditions. Nevertheless, how to extract meteorological parameters of high accuracy along schemes of transmission lines is not yet solved. The biggest challenge is to execute automatic extraction and comprehensive analysis of a variety of complex meteorological parameters. At present, designers can only have a rough estimation of these meteorological parameters. However, the precision and efficiency of the estimation can hardly meet requirements. This paper proposes an algorithm which can precisely extract and analyze multiple complex meteorological parameters along transmission lines. By means of GIS-based spatial reasoning, the algorithm analyzes and handles spatial relations between transmission lines and meteorological parameters, providing a solid foundation for the design of transmission lines.

\section{GIS-BASED SPATIAL REASONING}

Originated in the early 1970s, the theory of spatial reasoning has been developed quite rapidly in recent years[3]. Its research areas have expanded from artificial intelligence to many other areas, and GIS has become one of the most mature and most widely used areas. Spatial reasoning refers to a procedure of spatial object modeling, description and presentation, while also, accordingly, having qualitative or quantitative analysis of spatial relations between spatial objects using spatial theories and artificial intelligence. An important element of GIS-based spatial reasoning is geo-spatial reasoning, which refers to spatial reasoning that contains the knowledge of spatial relations[4]. Its key problem is how to use data stored in the database, combined with appropriate spatial constraints, to mine unknown spatial information. GIS-based spatial reasoning includes spatial reasoning of topological relations, direction relations, distance relations and combined relations. Spatial topological relations, direction relations and distance relations are three important aspects to describe spatial relations between spatial objects. There exist spatial topological relations as well as spatial direction relations and distance relations between spatial objects. These three kinds of relations are not completely independent but interrelated, interdependent and mutually restrictive. The perception of spatial relations between spatial objects also requires taking into account all these spatial relations. Reasoning integrating multiple spatial relations can obtain much more precise and abundant spatial information about spatial objects.

\section{EXTRACTION AND ANALYSIS OF METEOROLOGICAL PARAMETERS}

At preliminary design stage, after the completion of an initial scheme for a transmission line, designers need meteorological information along the scheme in order to verify the rationality of the scheme and make configurations for antipollution levels, anti-icing thicknesses and so forth of the transmission line afterwards. For example, as to pollution levels and icing thicknesses, segments (neighbor ones have different pollution levels or icing thicknesses unless the 
demarcation is a corner point, while all points of one segment have the same pollution level and icing thickness), along with accurate pollution level, icing thickness, start point, end point, start point name and end point name of each segment are all required.

\section{A. Data Structure}

Data structures used in the algorithm were named Node, ThematicInfo and LineSegment, as shown in figure 1. Class Node was designed to represent a point (corner point and intersection point) on a route and included properties like $\mathrm{X}, \mathrm{Y}$, name, distance to the start point along the route (cumulative distance), distance to the nearest corner point and so on. Class LineSegment was designed to indicate a segment of the route and included properties like start point, end point, list of meteorological parameters and so on. Class ThematicInfo was designed to represent meteorological parameters and included properties like meteorological thematic map name, list of parameters and list of parameters values.

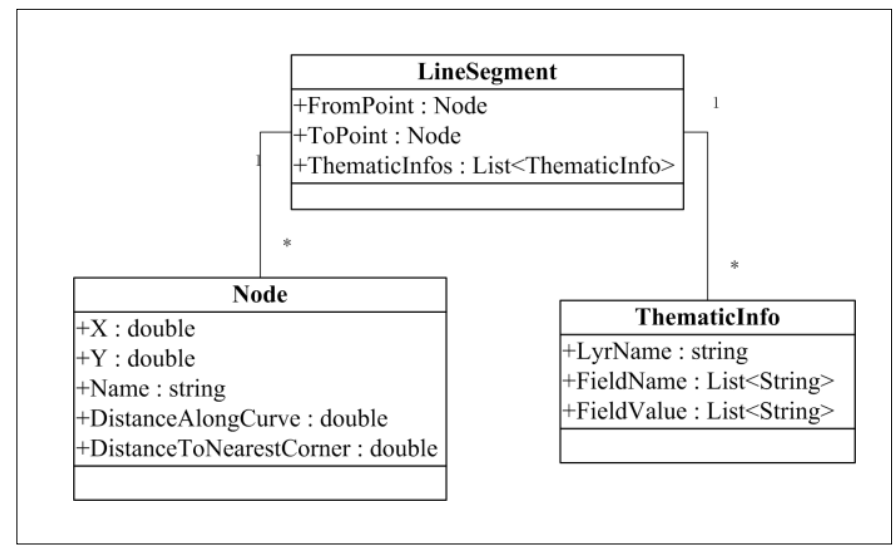

Fig. 1. Data structure.

Corner points of the route were named J0, J1, J2 and so on. Intersection points between the route and meteorological thematic maps were named using the distance to their nearest corner points. For example, there was an intersection between $\mathrm{J} 0$ and $\mathrm{J} 1$, if its distance to $\mathrm{J} 0$ was $8 \mathrm{~km}$ and to $\mathrm{J} 1$ was $7 \mathrm{~km}$, then the intersection would be named "J1-7km". Otherwise, if its distance to $\mathrm{J} 0$ was $6 \mathrm{~km}$ and to $\mathrm{J} 1$ was $9 \mathrm{~km}$, then the intersection would be named "J0+6km".

\section{B. Extraction of Single Meteorological Parameter}

Firstly, intersection points between the route and the meteorological map were calculated according to their topological relations. Secondly, the cumulative distance of every corner point and intersection point were calculated. Thirdly, all corner points and intersections were merged and sorted in accordance with their cumulative distances. Fourthly, distances to nearest corner points and names of all intersections were calculated. Fifthly, segments were generated using every two adjacent points from the point set. Finally, meteorological parameter values were calculated for all segments using GISbased spatial reasoning model[5]. The procedure which extracts single meteorological parameter along a route is shown in figure 2 .

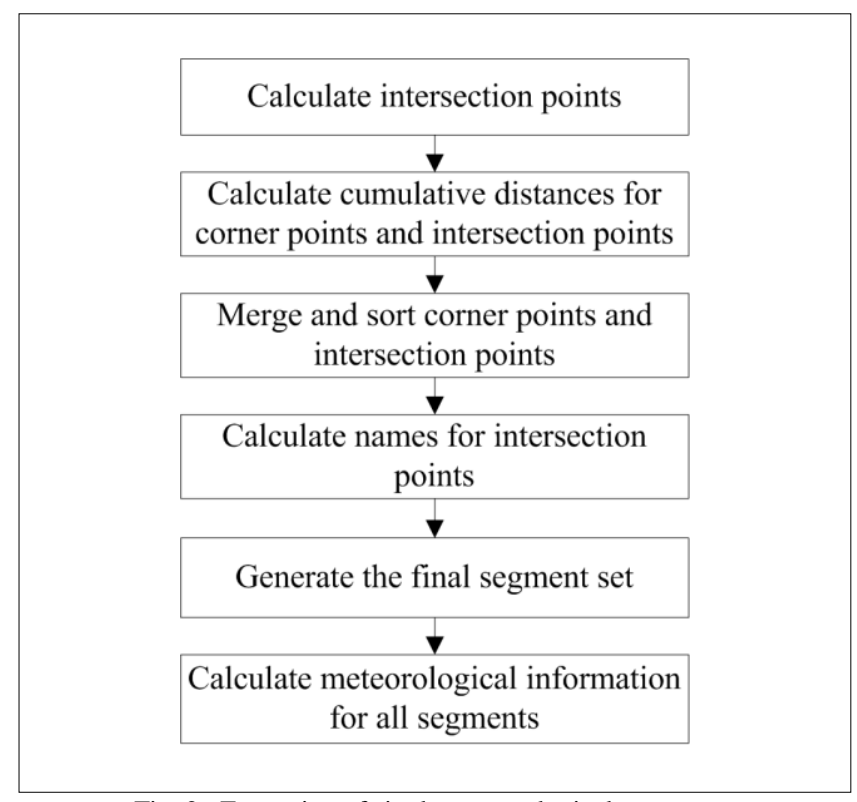

Fig. 2. Extraction of single meteorological parameter.

\section{Extraction of Multiple Meteorological Parameters}

After getting every single meteorological parameter value along the route, we need to merge these solitary results and derive segments of which neighbor ones had at least one different meteorological parameter value unless the demarcation was a corner point, while all points of one segment had the same meteorological parameters values. For example, between corner point $\mathrm{J} 0$ and $\mathrm{J} 1$, if for pollution level parameter, segment [J0,J0+400] had level I pollution and segment $[\mathrm{J} 0+400, \mathrm{~J} 1]$ had level II pollution, while for icing thickness parameter, segment [J0,J0+600] had 3mm icing and segment [J0+600,J1] had $5 \mathrm{~mm}$ icing, then the final results should be that segment [J0,J0+400] had level I pollution and $3 \mathrm{~mm}$ icing, segment [J0+400,J0+600] had level II pollution and $3 \mathrm{~mm}$ icing, and segment [J0+600, J1] had level II pollution and $5 \mathrm{~mm}$ icing.

In order for a comprehensive analysis of multiple meteorological parameters along the route, firstly, the final result point set of every single extraction results was gathered and merged. And all points were sorted on the accumulative distance. Points with the same accumulative distance were removed with only one left, and at this time we got the final point set. Secondly, the final result segment set of every single extraction results was gathered and merged. Each segment was represented by its starting point and therefore, each point in the final point set was corresponding to one or more segments. Segments with the same starting point were removed with only one left, however, all meteorological information of the removed segments need to be added to the left one. Now we got the intermediate segment set. Thirdly, with the first segment in the intermediate segment set, which had all meteorological information, as a benchmark, all segments were traversed. For those segments, which missed some 
meteorological information, we traversed backward until we found the first segment, which contained one of its missing meteorological parameters, and assigned the meteorological parameter to the segment, which lacked the parameter. We continued this procedure until all segments' meteorological information was complete. Fourthly, the final segment set was generated from the point set. Fifthly, we set and sorted meteorological information of the final segment set using the intermediate one. At this moment, we had got complete and accurate meteorological information along the route. Based on single meteorological parameter along a transmission line, the procedure which can extract multiple meteorological parameters along the transmission line is shown in figure 3.

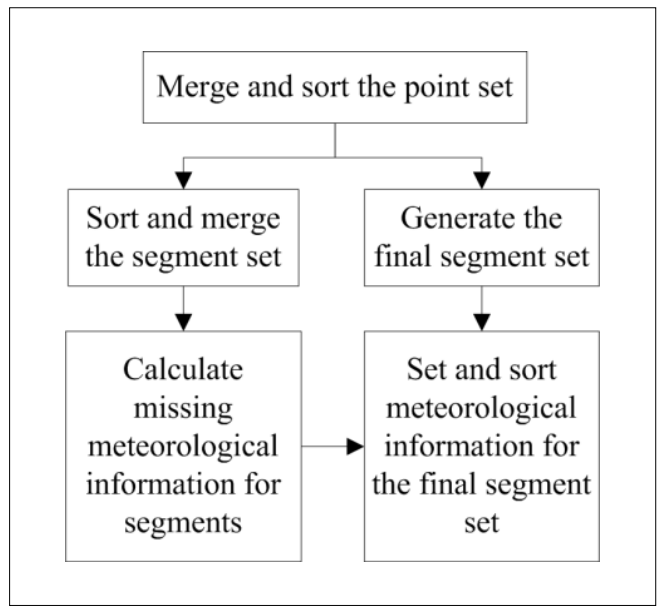

Fig. 3. Extraction of multiple meteorological parameters.

\section{EXPERIMENT RESULTS AND DISCUSSION}

This paper chose two meteorological thematic maps, which were icing thematic map and pollution thematic map, from the same area as shown in figure 4 . The red line represents a transmission line, which is up to $162.314 \mathrm{~km}$, and contains 52 corner points, which were identified as $\mathrm{J} 0, \mathrm{~J} 1$...J51 in the algorithm. The blue line represents icing data, which contains six thickness levels, namely [0mm,5mm], [5mm,10mm], $[10 \mathrm{~mm}, 15 \mathrm{~mm}],[15 \mathrm{~mm}, 20 \mathrm{~mm}],[20 \mathrm{~mm}, 30 \mathrm{~mm}]$ and $[30 \mathrm{~mm}$, $\infty]$. The green line represents pollution data, which contains two levels, namely level II and level III.

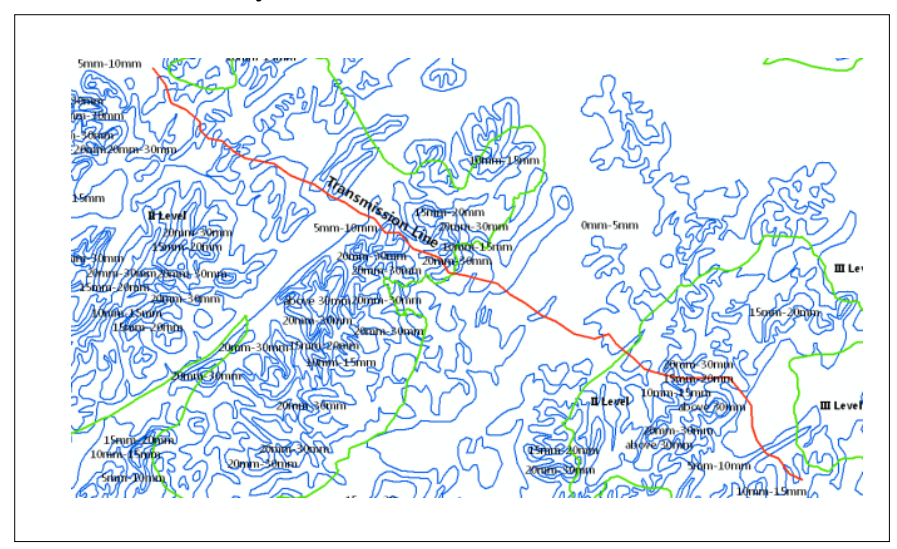

Fig. 4. Experiment data.
Using the algorithm proposed in this paper, firstly we extracted respective distribution of icing thicknesses and pollution levels along the route. And then we conducted a comprehensive analysis on the results. In the end, we got the overall distribution of icing thicknesses and pollution levels along the route, as shown in figure 5. The results show the segments, of which neighbor ones have different icing thicknesses or pollution levels unless the demarcation is a corner point, while all points of one segment have the same icing thickness and pollution level. In case the segments are too scattered, the algorithm supports merging operation of segments.

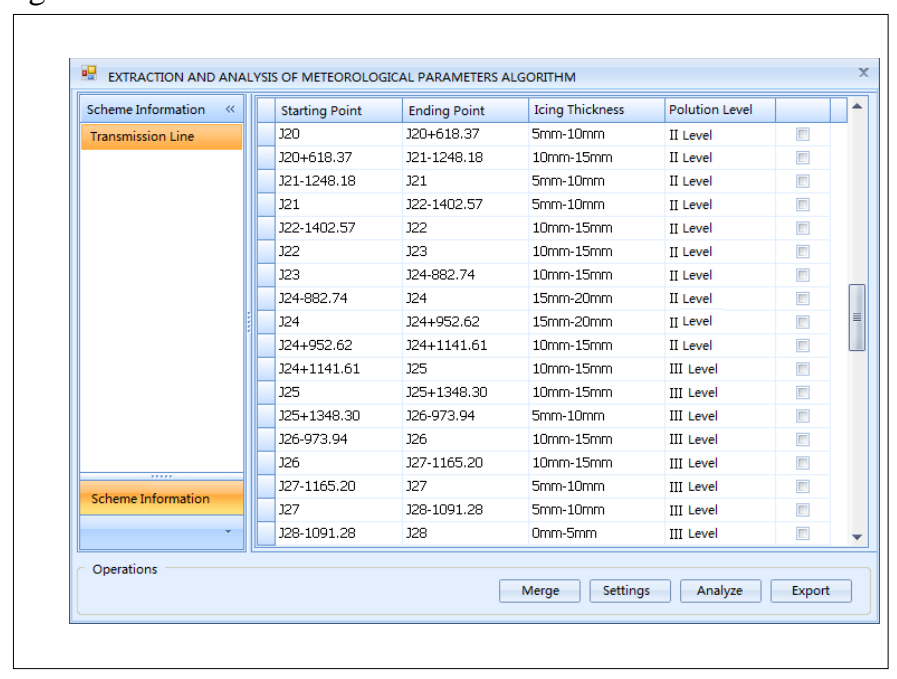

Fig. 5. Experiment results.

The results provide a basis for designers to make configurations for anti-pollution levels and anti-icing thicknesses of different sections of the transmission line. The entire calculation process took less than two seconds. In addition, we chose randomly 200 points along the route, and had a comparative analysis of the icing thickness value and pollution level value of each point in the thematic maps with the calculation results. And we concluded that the accuracy of the algorithm was $100 \%$, much higher than the artificial estimation accuracy.

\section{CONCLUSIONS}

Currently, China's acceleration in infrastructure construction has aggravated atmospheric pollution. Besides, bad weather occurs frequently in some area. As a result, accidents, such as pollution flashover and excessive icing, occur now and then. China's electric power departments have attached great importance to transmission line problems like anti-pollution flashover and anti-icing. Precautions concerning transmission line design, operation and maintenance have been made to prevent large area of pollution flashover and excessive icing. This paper proposes a solution for automatic extraction and comprehensive analysis of multiple complex meteorological parameters along transmission lines, and the results proved to be of high efficiency, accuracy and reliability. 


\section{REFERENCES}

[1] Song, L., Zhang, F., Liang, X., and Liao, Y. "Theoretical analysis on pollution flashover voltage prediction based on leakage current of porcelain insulator in service," Atlantis Press, January 2013.

[2] Zhuang, J. D., Zeng, W. L., Liu, P., Dai, W. X., and Liu, Q. "Application of photo-induced Nano-TiO2 film to anti-pollution flashover of ceramic insulator," Materials for Mechanical Engineering, vol. 36, pp. 85-88, 2012.
[3] Cohn, Anthony G., and Shyamanta M. Hazarika. "Qualitative spatial representation and reasoning: an overview," Fundamenta Informaticae, vol. 46, pp. 1-29, January 2001.

[4] Longley, P., Goodchild, M. F., Maguire, D., and Rhind, D. "Geographic information systems and science," Wiley,2005.

[5] Egenhofer, Max. "Reasoning about binary topological relations," Advances in Spatial Databases, vol. 525, pp. 141-160, 1991. 